\title{
Elementos traza y trastorno afectivo bipolar
} Trace Elements and Bipolar Affective Disorder

\author{
Kerly Viviana Cruz \\ Pontificia Universidad Javeriana, Colombia \\ Mario Benavides-Solarte \\ Universidad Militar Nueva Granada, Colombia \\ Carlos Gómez-Restrepo \\ Pontificia Universidad Javeriana, Colombia
}

Fecha de recepción: 04/09/2016 | Fecha de aprobación: 15/03/2017 | 18/01/2017

Cómo citar: Cruz KV, Benavides-Solarte M, GómezRestrepo C. Elementos traza y trastorno afectivo bipolar. Univ Med. 2017;58(2):1-8. doi: https://doi.or g/10.11144/Javeriana.umed58-2.traz

Conflictos de interés: Los autores declaran no tener ningún tipo de conflicto de interés con relación a este estudio.

\section{RESUMEN}

Introducción: Los elementos traza u oligoelementos son micronutrientes disponibles en mínima cantidad en el cuerpo humano y constituyen funciones vitales, lo que les confiere su importancia. El exceso o déficit en las concentraciones de oligoelementos como zinc, litio, cobre y plomo podrían estar involucrados en las diferentes fases del trastorno afectivo bipolar. Objetivo: Analizar la información actual disponible en la literatura sobre la relación entre elementos traza y el trastorno afectivo bipolar. Métodos: Búsqueda en paralelo por dos revisores en las bases de datos Ovid, Pubmed, SciELO y Cochrane. Resultados: Se encontraron un total de 47 artículos publicados entre 1988 y 2015, aunque al final se evaluaron 12 artículos en texto completo. Conclusiones: La literatura disponible sugiere asociación entre altas cantidades de zinc en fase maniaca y escasa presencia en la fase depresiva de pacientes con trastorno afectivo bipolar. No se encontró asociación significativa con los otros oligoelementos.

Palabras clave

trastorno afectivo bipolar; elementos traza; litio; zinc.

\begin{abstract}
Introduction: Trace elements or trace mineral are dietary minerals found in few amounts in the human body, they play important roles in vital functions, which gives them their significance. Excess or deficit levels of trace elements such as zinc, lithium, copper and lead may be involved in the development of bipolar disease; There is a possible relation among imbalances of trace elements and the stages of Bipolar Disease. Methods: Parallel search made by two reviewers on the data basis of Ovid, Pubmed, SciELO, and Cochrane. Results: A total of 47 articles published between 1998-2015 were found, finally 12 full text articles were evaluated. Conclusions: Available literatures suggest an association among elevated levels of zinc in mania and low levels in depressive phase in patients with bipolar disorder. No significant association with other trace elements was found. Further studies are required with larger numbers of patients to establish these associations.
\end{abstract}

Keywords

bipolar affective disorder; trace elements; lithium; zinc. 


\section{Introducción}

En las últimas décadas se han propuesto múltiples hipótesis sobre la etiología y la fisiopatología del trastorno afectivo bipolar (TAB) [1]. Se ha descrito una posible asociación entre la alteración de los elementos traza y la presentación de la enfermedad bipolar [2]. Sin embargo, la información disponible en la literatura es escasa. De acuerdo con el estudio nacional de salud mental realizado en el 2003 en Colombia y datos reportados por la Organización Mundial de la Salud, los trastornos del afecto se presentan en el 0,9\% de la población y se ha observado una edad similar de presentación en TAB I, TAB II y depresión (entre 20 y 24 años) $[1,3,4,5,6]$. Según el Informe de carga global de enfermedad de la Organización Mundial de la Salud, del 2000, el TAB ocupa uno de los primeros lugares como causa de discapacidad. Se atribuyen a las enfermedades mentales y neurológicas hasta el 30,8 \% de los años perdidos por discapacidad $[1,3,4,5]$.

Los elementos traza u oligoelementos son micronutrientes presentes en el cuerpo humano en un porcentaje mínimo con importantes funciones: hacen parte de algunas enzimas, inducen expresión genética, controlan procesos biológicos, entre otras funciones vitales. Se ha documentado en múltiples estudios la relación existente entre elementos traza y las enfermedades de mayor impacto alrededor del mundo, como cardiopatía isquémica, accidente cerebrovascular, hipertensión arterial, diabetes mellitus [6]. También se han asociado con diferentes enfermedades mentales como el TAB [7].

Los elementos traza son minerales de la dieta que se encuentran en cantidades mínimas, pues ocupan menos del 0,02\% del peso corporal total [8]. Tienen múltiples funciones vitales, lo que les confiere su importancia [9]. Se han descrito seis elementos traza esenciales: cobre, cromo, zinc, selenio, molibdeno y yodo, cuyo requerimiento diario oscila entre $50 \mu \mathrm{g}-18 \mathrm{mg}$ [9]. Existen, además, elementos potencialmente tóxicos que parecen estar involucrados en funciones esenciales y su exceso puede ser un agravante de la salud [10]. Las alteraciones de estos oligoelementos se asocian con diferentes enfermedades neuropsiquiátricas, y entre los más estudiados se encuentran zinc, cobre, litio, plomo $[10,11,12]$. En la depresión mayor se ha demostrado bajas concentraciones de elementos traza [3], lo que se puede estar en relación con los episodios depresivos que presenta la enfermedad bipolar $[4,13]$.

El TAB tiene una etiología multifactorial: estrés social, factores genéticos y alteraciones bioquímicas o anatómicas en sistema nervioso central. McGuffin et al., citados en [14], demostraron que hasta el $85 \%$ de los pacientes presenta antecedentes familiares. Diferentes pruebas genéticas post mortem, estudios bioquímicos y de imagen apuntan hacia un papel importante en la desregulación de glutamato y receptor $\mathrm{N}$-metil-D-aspartato (NMDAR) en la etiopatogenia del TAB durante la manía y la depresión [15]. Se han encontrado niveles alterados de glutamato, además de anormalidades en la expresión de genes, que también afectan la expresión y funciones de NMDAR en pacientes con TAB [10]. Un exceso de glutamato y una posterior sobrestimulación de NMDAR, se supone, es uno de los mediadores más importantes de los procesos de excitotoxicidad y muerte neuronal a través de un exceso de afluencia de calcio a la célula [16].

Aparte de lo anterior, en el TAB se asocia con una disfunción mitocondrial en lo referente a disminución de procesos de respiración, cambios en su morfología, alteraciones del ADN mitocondrial, dado por un incremento de polimorfismos, mutaciones y baja regulación de las moléculas del ADN mitocondrial y de las proteínas relacionadas con procesos de respiración [11]. El daño oxidativo puede precipitar disfunción mitocondrial, y esto se ha documentado en diferentes trastornos psiquiátricos [12].

El TAB incluye distintas dimensiones del ser humano que afectan de manera significativa la calidad de vida, las relaciones interpersonales, la salud mental y, desde el punto de vista económico, disminuye la productividad y lleva a pérdida del empleo [13]. Lo anterior influye 
en la salud pública y demanda un diagnóstico y manejo oportuno. El amplio espectro de factores implicados en la presentación del TAB, además del impacto individual y en salud pública, hace necesario una mayor comprensión de los aspectos fisiopatológicos, con el fin de definir nuevas maneras de abordar clínicamente a estos pacientes [5]. Debido a esto, el objetivo de la presente revisión es actualizar la información disponible en la literatura sobre la relación entre elementos traza y el TAB.

\section{Métodos}

Para la localización de los documentos bibliográficos se utilizaron diferentes bases de datos: Ovid, Pubmed, SciELO y Cochrane. La búsqueda se realizó en paralelo por dos revisores con conocimientos del área de la salud, a fin de que la extracción de la información se llevara a cabo de manera pareada. La búsqueda utilizó los siguientes términos $\mathrm{MeSH}$ : trace elements, oligoelements, bipolar disorder y affective disorder. Y los términos libres: manic mood, zinc, lithium, copper, cadmium, aluminium, phosphorus, magnesium, mania e hypomania. La información se limitó a estudios llevados a cabo en humanos. Se incluyeron artículos en inglés y español.

De ese modo, se encontraron 47 artículos publicados entre 1988 y 2015, y de estos se excluyeron 29, tras la eliminación de duplicados, artículos en formato de póster o estudios que no profundizaban en la enfermedad bipolar o sobre elementos traza. De las 18 referencias que se escogieron por títulos se excluyeron otros 6 artículos que no pudieron encontrarse en formato de texto completo. Al final se evaluaron 12 artículos en texto completo (figura 1).
Figura 1. Diagrama de flujo

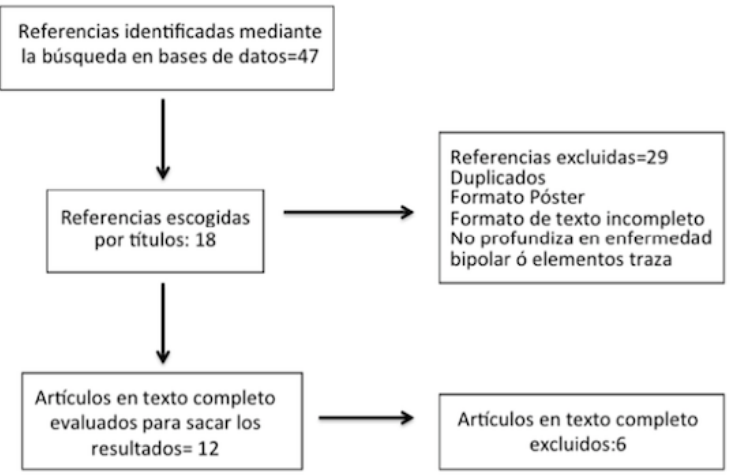

\section{Resultados}

De los elementos traza que se mencionan en la literatura se encontraron 7 artículos que mencionan el zinc; 5, el cobre; 6, el litio, y 2, el plomo y el cadmio (tabla 1 ).

Tabla 1. Artículos incluidos

\begin{tabular}{|c|c|c|c|}
\hline Título & Autores & Año & Revista \\
\hline $\begin{array}{l}\text { Trace elemental distribution in the scalp hair of } \\
\text { bipolars using PIXE technique }\end{array}$ & Pradeep et al. & 2014 & Medical Hypotheses \\
\hline Trace lithium in mood disorders & Dafflon et al. & 1998 & $\begin{array}{l}\text { Journal of Affective } \\
\text { Disorders }\end{array}$ \\
\hline $\begin{array}{l}\text { Decreased serum zinc concentration during } \\
\text { depressive episode in patients with bipolar disorder }\end{array}$ & Siwek et al. & 2015 & $\begin{array}{l}\text { Journal of Affective } \\
\text { Disorders }\end{array}$ \\
\hline Trace elements in bipolar disorder & $\begin{array}{l}\text { González- } \\
\text { Estecha et al. }\end{array}$ & 2011 & $\begin{array}{l}\text { Journal of Trace } \\
\text { Elements in Medicine } \\
\text { and Biology }\end{array}$ \\
\hline Zinc as a marker of affective disorders & Siwek et al. & 2013 & $\begin{array}{l}\text { Pharmacological } \\
\text { Reports }\end{array}$ \\
\hline Bipolar disorders and Wilson's disease & Carta et al. & 2012 & BMC Psychiatry \\
\hline $\begin{array}{l}\text { Neocortical levels of lithium are increased in bipolar } \\
\text { disorder }\end{array}$ & Friedlich & 2012 & Molecular Psychiatry \\
\hline $\begin{array}{l}\text { Trace elements and the electroencephalogram during } \\
\text { long-term lithium treatment }\end{array}$ & Harvey et al. & 1992 & $\begin{array}{l}\text { British Journal of } \\
\text { Phsichiatry }\end{array}$ \\
\hline Is lithium potentially a trace element? & Terao & 2015 & $\begin{array}{l}\text { World Journal Of } \\
\text { Phsichiatry }\end{array}$ \\
\hline $\begin{array}{l}\text { Assessment of serum macro and trace element } \\
\text { homeostasis and the complexity of inter-element } \\
\text { relations in bipolar mood disorders }\end{array}$ & Mustak et al. & 2008 & Clinica Chimica Acta \\
\hline $\begin{array}{l}\text { Associations of serum } \mathrm{Ca} \text { and } \mathrm{Mg} \text { levels with mental } \\
\text { health in adult women without psychiatric disorder }\end{array}$ & Jung et al. & 2010 & $\begin{array}{l}\text { Biological Trace } \\
\text { Element }\end{array}$ \\
\hline $\begin{array}{l}\text { Trace lithium is inversely associated with male } \\
\text { suicide after adjustment of climatic factors }\end{array}$ & Shiotsuki et al. & 2016 & $\begin{array}{l}\text { Journal of Affective } \\
\text { Disorders }\end{array}$ \\
\hline
\end{tabular}

Aproximación nosológica del trastorno afectivo bipolar

El TAB es catalogado por el Manual diagnóstico y estadístico de los trastornos mentales (DMS-5) como un grupo heterogéneo de trastornos del estado de ánimo que incluye el TAB I, el TAB II, episodios mixtos y una categoría diagnóstica aparte, la ciclotimia [14]. Cada uno de estos grupos enmarca ciertas características que los diferencian entre ellos. El TAB tipo 
I se caracteriza por la presencia de episodios maniacos francos, y en el TAB tipo II el paciente experimenta síntomas de manía que no cumplen todos los criterios de un episodio maniaco. Esto se conoce como hipomanía. Por otro lado, la ciclotimia se caracteriza por la presencia de episodios recurrentes de hipomanía y depresión subclínica [13].

El $\mathrm{TAB}$ es reconocido por los síntomas maniacos, que conforman la principal diferencia con el resto de enfermedades psiquiátricas. Sin embargo, entre el 12 y el $16 \%$ de estos pacientes no presenta episodios depresivos en el curso de su enfermedad, característica que los enmarca en el grupo de manía unipolar [15]. A pesar de los estudios que demuestran la existencia de la manía unipolar, el DSM-5 no reconoce la manía pura o la hipomanía, razón por la cual se incluyen en el grupo del TAB [17]. Los episodios maniacos están presentes aproximadamente el $10 \%$ del tiempo; mientras que los síntomas depresivos corresponden a un $40 \%$, siendo este el de mayor presentación [15]. La manía ha sido el causal de efectos devastadores, sobre todo en el ámbito social y económico de estos pacientes y sus familias. No obstante, durante el episodio depresivo surgen las ideas suicidas y se consuman los suicidios [18], de manera que los dos polos de la enfermedad definen el impacto en salud pública. Durante estas fases del TAB se han medido las concentraciones de elementos traza, como zinc y cobre y se han encontrado alteraciones $[7,10]$.

Asociación de elementos traza y enfermedad bipolar

Desde los años ochenta, los elementos traza han adquirido gran importancia en el campo de la salud mental y se han documentado concentraciones alteradas en diferentes enfermedades, incluido el TAB. Entre los micronutrientes más estudiados se encuentran el zinc, el cobre, el litio, el cadmio, el aluminio, el magnesio, el fósforo, entre otros $[11,19,20]$, de los cuales se realizan mediciones en sangre, orina y muestras de cabello [21], mediante espectrometría de absorción atómica y otros métodos tradicionales [17].

Zinc

El zinc es un elemento esencial para humanos y animales. La cantidad de zinc en el cuerpo está alrededor de $40 \mathrm{mg}$ por kilogramo y el funcionamiento de muchas enzimas requieren su participación (alrededor de 100): metabolismo de proteínas y ácidos nucleicos, funcionamiento adecuado del sistema inmune, procesos de cicatrización de las heridas, procesos de percepciones del gusto y el olfato y en la síntesis del ADN [7,22,23].

El déficit de zinc puede dar lugar a alteraciones inmunitarias, facilita procesos infecciosos, condiciona enfermedad diarreica, lesiones cutáneas como erupciones dérmicas, además de problemas neuropsiquiátricos [22]. Cambios en la homeostasis de zinc en el cerebro se han asociado con alteraciones comportamentales, problemas de aprendizaje, alteraciones de la función cognitiva y ciertos trastornos neurológicos. Se ha documentado asociación entre concentraciones de zinc y episodios depresivos con un posible papel en los mecanismos fisiopatológicos que determinan esta condición. La actividad antidepresiva del zinc se debe a su acción como antagonista del NMDAR [20]. Algunos estudios sugieren un efecto antidepresivo mediante la inhibición de la enzima glucógeno-sintasa-cinasa3B [24].

En el 2010, Monserrat González-Estecha et al. [10] midieron las concentraciones de zinc en pacientes con $\mathrm{TAB}$ y documentaron cantidades elevadas en pacientes en fase maniaca en comparación con aquellos en fase depresiva.

Ole Grönli et al. [18] compararon en el 2013 la prevalencia de déficit de zinc en pacientes geriátricos con depresión y otros diagnósticos psiquiátricos. Concluyeron que existe una deficiencia clara de zinc en este grupo etario; sin embargo, no hay una asociación clara entre el diagnóstico de depresión y este elemento traza. Asimismo, en la revisión publicada por Marcin Siwek et al. [24] se 
sugiere el papel modulador del zinc en la acción de neurotransmisores excitatorios en sistema nervioso central, al proponerlo como un marcador de trastornos del afecto. En pacientes depresivos se documenta, además, una disminución de zinc en relación con una respuesta inflamatoria de fase aguda, con estrés oxidativo, niveles bajos de transferrina, proteínas, albúmina y niveles elevados de interleucina (IL-6), linfocitos T CD4/CD8 y neopterina [25].

\section{Cobre}

El cobre es un elemento traza necesario para los procesos de crecimiento y desarrollo en seres humanos. Hace parte de metaloenzimas y participa en diferentes vías biológicas como la de las catecolaminas, monoaminooxidasa, B-hidroxilasa y tirosina hidroxilasa; además, se relaciona con los procesos de formación de mielina y eritropoyetina con un efecto antioxidante, que son los mecanismos relacionados con la modulación del afecto [29].

En el trabajo publicado por Monserrat González-Estecha et al. [10] en el que se midieron las concentraciones séricas de cobre y zinc mediante espectrometría de absorción atómica en pacientes con diagnóstico de TAB, no se encontró diferencia significativa en las cantidades de cobre entre el grupo de pacientes y controles [8].

Mohhamed S. Mustak et al. [13] documentaron concentraciones elevadas de cobre en pacientes con $\mathrm{TAB}$ en fase maniaca y depresiva, comparadas con los controles, al igual que concentraciones superiores de otros elementos traza en pacientes con diagnóstico de TAB. En pacientes con enfermedad de Wilson se describen fases maniacas y depresivas, catatonia, agitación y agresión como manifestaciones iniciales de la enfermedad, que sugieren una asociación entre estos cambios de comportamiento y cantidades elevadas de cobre; pero no se cuenta con evidencia sólida que demuestre esta asociación [26].
Litio

El litio se ha utilizado como estabilizador del estado de diferentes trastornos (por ejemplo, el $\mathrm{TAB}$ ), que ha mostrado alta eficacia en pacientes con depresión recurrente y esquizofrenia [22]. El cloruro de litio bloquea la degradación de IP3, a nivel del inositol-4-fosfato (IP1) y rompe con ello el ciclo del fosfatoinositol. En enfermedades maniaco-depresivas se produce una activación de los canales de calcio sensibles a IP3 y ello da lugar a mayor disponibilidad de calcio citosólico. Su capacidad para estabilizar o prevenir cambios en el estado de ánimo sugiere su acción en la etiopatogenia del TAB [22,28].

Se ha descrito, además, que la administración de microdosis de litio tiene efectos antidemencia; sin embargo, no hay hallazgos que respalden el uso de estas mismas concentraciones ante psicosis o manía [22].

Desde 1990, Goodwin y Jamison [26] han sugerido hipótesis acerca de las anormalidades en la excitabilidad o en la conductancia iónica de la membrana celular en pacientes con TAB. En pacientes con trastornos afectivos se plantea una posible disfunción de membrana celular neuronal como condicionante en el intercambio de las concentraciones de litio; sin embargo, concentraciones terapéuticas de litio interfieren con la transducción de señales intracelulares neuronales. Desde 1995 se han desarrollado y validado métodos analíticos para determinar la concentración de litio en fluidos biológicos. Daffon et al. [26] midieron concentraciones de litio plasmático en orina de pacientes con $\mathrm{TAB}$ sin encontrar diferencias significativas al compararlos con los niveles documentados en el grupo control. Describieron cantidades aumentadas de litio en pacientes con alto consumo de medicamentos antiinflamatorios, frutas cítricas, cerveza y una niveles bajos en pacientes que consumían antidepresivos serotoninérgicos [22].

Sin embargo, las observaciones descritas al evaluar la neocorteza de cadáveres de pacientes con TAB documentan altas concentraciones de litio, lo que sugiere una respuesta compensatoria 
a la deficiencia de litio en su vía dependiente. Es decir, cantidades elevadas de litio pueden ser causadas por un secuestro de litio en la vía, con deficiencia secundaria de la biodisponibilidad de este micronutriente [24].

En síntesis, se infiere que el litio es un micronutriente esencial. Evidencia sólida confirma una relación estrecha en la modulación del estado de ánimo y el litio; de ahí su uso como estabilizador en el TAB. A pesar del déficit de estudios en este campo, se cree que la biodisponibilidad del litio es inversamente proporcional a su concentración en el plasma, lo que involucra este elemento en la etiopatogenia de la enfermedad bipolar [30].

\section{Plomo y cadmio}

El plomo se considera un elemento neurotóxico ubicuo en el medio ambiente. Probablemente, no exista una medida segura de este elemento en la sangre. La exposición a plomo puede desencadenar problemas psiquiátricos, como depresión, ansiedad o trastorno de pánico.

Se considera la exposición al humo de tabaco y a ciertos alimentos, como las principales fuentes de cadmio; por esta razón se asocia fuertemente con enfermedad cardiovascular, específicamente infarto agudo de miocardio y enfermedad arterial periférica.

Pocos estudios involucran análisis de las concentraciones de plomo o cadmio en la etiopatogenia del $\mathrm{TAB}$, dado que estas pueden aumentar por las diferentes sustancias psicoactivas consumidas por este grupo de pacientes [28].

Montserrat González-Estecha et al. [10] reportaron un aumento de las concentraciones de plomo en sangre y orina de pacientes con $\mathrm{TAB}$, además de cadmio elevado en la orina, comparado con el grupo control. Estos hallazgos sugieren una relación de los valores hallados de estos elementos traza con estrés oxidativo, que es uno de los factores condicionantes del desarrollo del TAB.

En definitiva, la literatura reporta un aumento en las cantidades de plomo y cadmio en pacientes con $\mathrm{TAB}$, sugiere concentraciones que recientemente han sido asociadas al deterioro de la función cognitiva y a la enfermedad cardiovascular. Pese a estas investigaciones, no existe suficiente evidencia que respalde los hallazgos encontrados.

\section{Conclusiones}

Los elementos traza son minerales de la dieta que se encuentran en cantidades mínimas y ocupan menos del 0,02\% del peso corporal total. Son de utilidad en múltiples procesos biológicos. La literatura mundial publicada describe la relación existente entre sus niveles y diferentes trastornos psiquiátricos, incluido el $\mathrm{TAB}$, y se han encontrado estudios que sugieren cantidades elevadas de zinc en pacientes con TAB en fase maniaca y bajas en pacientes en fase depresiva $[18,27]$; pero no se cuenta con estudios que establezcan relación existente entre las diferentes fases del TAB y litio, cobre, plomo y cadmio. No existe por el momento evidencia acerca de la relación entre las concentraciones de elementos traza y TAB. Se necesitan más estudios con mayor número de pacientes para establecer con claridad esta posible relación.

\section{Referencias}

1. Pradeep AS, Naga Raju GJ, Sattar SA, Sarita P, Prasada Rao AD, Ray DK, et al. Trace elemental distribution in the scalp hair of bipolars using PIXE technique. Med Hypotheses. 2014;82(4):470-7. doi: http:// dx.doi.org/10.1016/j.mehy.2014.01.028

2. Jung KI, Ock SM, Chung JH, Song CH. Associations of serum $\mathrm{Ca}$ and $\mathrm{Mg}$ levels with mental health in adult women without psychiatric disorders. Biol Trace Elem Res. 2010;133(2):153-61.

3. Bravo MF, Lahera G, Lalucat L, Fernández-Liria A. Guía de práctica clínica sobre el trastorno bipolar: tratamiento farmacológico y psicosocial. Med Clin (Barc) [internet]. 2013;141(7):305.e1-305.e10. Disponible 
en: http://www.sciencedirect.com/science/ article/pii/S0025775313003977

4. Ministerio de Salud y Protección Social. Análisis de situación de salud: Colombia, 2013 [internet]. Bogotá: Ministerio; 2014. Disponible en: https://www.minsalud.gov.co/sites/rid/ Lists/BibliotecaDigital/RIDE/VS/ED/PSP/ ASIS\%2024022014.pdf

5. World Health Organization. Global status report on noncommunicable diseases 2014 [internet]. 2014. Disponible en: http://apps.who.int/iris/bitstream/1066 5/148114/1/9789241564854_eng.pdf?ua= 1

6. Flores CR, Puga MP, Wrobel K, Garay Sevilla ME, Wrobel K. Trace elements status in diabetes mellitus type 2: Possible role of the interaction between molybdenum and copper in the progress of typical complications. Diabetes Res Clin Pract. 2011;91(3):333-41.

7. Natural Medicines. Bipolar disorder. 2016.

8. Mustak MS, Rao TSS, Shanmugavelu P, Sundar NMS, Menon RB, Rao RV, et al. Assessment of serum macro and trace element homeostasis and the complexity of inter-element relations in bipolar mood disorders. Clin Chim Acta. 2008;394(1-2):47-53.

9. Solomon DA, Leon AC, Coryell WH, Li C, Fiedorowicz JG, Boyken ML. NIH Public Access Duration of Mood Episodes. ArchGenPsychiatry. 2010;67(4):339-47.

10. González-Estecha M, Trasobares EM, Tajima K, Cano S, Fernández C, López JL, et al. Trace elements in bipolar disorder. J Trace Elem Med Biol. 2011;25(suppl. 1):78-83.

11. Clay HB, Sillivan S, Konradi C. Mitochondrial dysfunction and pathology in bipolar disorder and schizophrenia. Int J Dev Neurosci. 2011;29(3):311-24. doi: http://dx.doi.org/10.1016/j.ijdevneu.2 010.08.007

12. Ng F, Berk M, Dean O, Bush AI. Oxidative stress in psychiatric disorders: evidence base and therapeutic implications. Int J Neuropsychopharmacol.
2008;11(6):851-76. doi: $\quad 10.1017 /$ S1461145707008401

13. García-Blanco AC, Sierra P, Livianos L. Nosología, epidemiología y etiopatogenia del trastorno bipolar: últimas aproximaciones. Psiquiatr Biol. 2014;21(3):89-94.

14. Khouzam HR, Singh F. Bipolar disorder: historic perspective, current pharmacologic treatment options and a review of quetiapine. Expert Rev Neurother. 2006;6(2):131-44.

15. Shulman KI, Tohen M. Unipolar mania reconsidered: Evidence from an elderly cohort. $\mathrm{Br} \mathrm{J}$ Psychiatry. 1994;164(apr.):547-9.

16. Mitchell PB, Malhi GS. Bipolar depression: Phenomenological overview and clinical characteristics. Bipolar Disord. 2004;6(6):530-9.

17. Dafflon M, Décosterd LA, Biollaz J, Preisig M, Dufour $\mathrm{H}$, Buclin T. Trace lithium in mood disorders. J Affect Disord. 1999;54(1-2):199-203.

18. Grönli O, Kvamme JM, Friborg $\mathrm{O}$, Wynn R. Zinc deficiency is common in several psychiatric disorders. PLoS One. 2013;8(12):6-13.

19. Nowak G, Szewczyk B. Mechanisms contributing to antidepressant zinc actions. Pharmacol Reports. 2002;54(6):58792.

20. Paoletti P, Vergnano AM, Barbour B, Casado M. Zinc at glutamatergic synapses. Neuroscience. 2009;158(1):12636. doi: http://dx.doi.org/10.1016/j.neurosc ience.2008.01.061

21. Schöpfer J, Schrauzer GN. Lithium and other elements in scalp hair of residents of Tokyo Prefecture as investigational predictors of suicide risk. Biol Trace Elem Res. 2011;144(1-3):418-25.

22. Shiotsuki I, Terao T, Ishii N, Takeuchi S, Kuroda Y, Kohno K, et al. Trace lithium is inversely associated with male suicide after adjustment of climatic factors. J Affect Disord. 2016;189:282-6. doi: http://dx.doi. org/10.1016/j.jad.2015.09.070 
23. Friedlich AL. Neocortical levels of lithium are increased in bipolar disorder. Mol Psychiatry. 2012;17(1):3-4.

24. Siwek M, Sowa-Kućmab M, Styczeń K, Szewczyk B, Reczyński W, Misztak P, et al. Decreased serum zinc concentration during depressive episode in patients with bipolar disorder. J Affect Disord. 2016;190:272-7.

25. Siwek M, Szewczyk B, Dudek D, Styczeñ K, Sowa-Kuæma M, Mlyniec K, et al. Zinc as a marker of affective disorders. Pharmacol Reports. 2013;65(6):1512-8.

26. Carta MG, Sorbello O, Moro MF, Bhat KM, Demelia E, Serra A, et al. Bipolar disorders and Wilson's disease. BMC Psychiatry. 2012;12:53. doi: 10.1186/1471-244X-12-52.

27. Terao $T$. Is lithium potentially a trace element? World J Psychiatry. 2015;5(1):1-3.

28. Aliasgharpour M, Farzami M. Trace elements in human nutrition: A review. Int J Med Invest. 2013;2(3):115-28.

29. Wada O. What are Trace Elements\#? Their deficiency and excess states. JPN Med Assoc J. 2004;47(5):351.

30. Vasudevaraju P, Bharathi TJ, Shamasundar NM, Subba Rao K, Balaraj BM, et al. New evidence on iron, copper accumulation and zinc depletion and its correlation with DNA integrity in aging human brain regions. Indian J Psychiatry. 2010;52(2):140-4. 